\title{
On general filtering problem of stationary processes with fixed transformation
}

Long suo Li

Correspondence:

lilongsu06982@126.com

Department of Mathematics,

Harbin Institute of Technology,

Harbin 150001, P.R.China

\begin{abstract}
A fixed transformation are given for one-dimensional stationary processes in this paper. Based on this, we propose a general filtering problem of stationary processes with fixed transformation. Finally, on a stationary processes with no any additional conditions, we get the spectral characteristics of $P_{H_{\eta}(t)} \xi$ in the space $L^{2}\left(F_{X}(d \lambda)\right)$, and then we calculate the value of the best predict quantity $Q$ of the general filtering problem.
\end{abstract}

Keywords: stationary processes, fixed transformation, fillering

\section{Introduction}

The Prediction theory is an important part of stationary processes, also linear filter problems is an important part of Prediction theory. The linear filtering problem of multidimensional stationary sequence and processes for a linear system are firsted studied by Rosanov in [1], and then a series of general filter problem of stationary process for a linear system are studied in [2-9]. Theoretically, this problem is a extend of the classic prediction problem. But it has high practical value, it also widely applied in communication, exploration, space technology and automatic control, etc.

\section{Propose the problem}

Let $X(t), t \in \mathbf{R}$ be (simple) wide stationary process. Let

$$
\begin{gathered}
H_{X}=\mathcal{L}\{X(t), t \in \mathbf{R}\} \\
H_{X}(t)=\mathcal{L}\{X(s), s \leq t, s \in \mathbf{R}\}
\end{gathered}
$$

Suppose the complex-value function $b(t)$ statisfing the following conditions

1)

$$
b(t) \in L^{\prime}[0,+\infty) \cap L^{2}[0,+\infty) \quad(2-1)
$$

2)

$$
b(t) \quad \text { for } t<0
$$

Let

$$
B(\lambda)=\int_{0}^{\infty} b(t) e^{-i \lambda t} d t
$$

(c) $2011 \mathrm{Li}$; licensee Springer. This is an Open Access article distributed under the terms of the Creative Commons Attribution License (http://creativecommons.org/licenses/by/2.0), which permits unrestricted use, distribution, and reproduction in any medium, provided the original work is properly cited. 
then $B(\lambda)$ is boundary values analytic function $B(z)$ in the low half plane

We can obtain

$$
B(\lambda) \neq 0, \quad \text { a.e. } \quad \text { Leb }
$$

Let

$$
E=\{\lambda: B(\lambda)=0\}, \bar{E}=(-\infty, \infty)-E
$$

then

$$
L(E)=0
$$

Let

$$
\eta(t)=\int_{0}^{\infty} b(s) \cdot X(t-s) d s=\int_{0}^{\infty} b(s) U^{-s} X(t) d s
$$

where $U$ is the shift oprator of $X(t)$ in the space $H_{X}$.

Then, for each $\xi \in H_{X}$

1) Find out the value of $Q$,

$$
Q=\inf _{\tilde{\xi} \in H_{X}(t)}\left\|\int_{0}^{\infty} b(s) U^{-s} \widetilde{\xi} d s-\widetilde{\xi}\right\|^{2}
$$

2) Then, we will prove that

$$
Q=\left\|P_{H_{\eta}(t)} \xi-\xi\right\|^{2}
$$

and solve the spectral characteristics of $P_{H_{\eta}(t)}$ in the space $L^{2}\left(F_{X}(d \lambda)\right)$.

\section{Main result}

Let the random spectral measure of $X(t)$ is $\Phi_{X}(d \lambda)$, and the spectral measure is $F_{X}$ $(d \lambda)$, the broad spectral measure which also named the spectral measure of absolutely continuous part of $F$ is $f_{X}(\lambda)$.

The Lebesgue decomposition of $F_{X}(d \lambda)$ is

$$
F_{X}(d \lambda)=f_{X}(\lambda) d \lambda+\delta(d \lambda)
$$

where the Lebesgue measure of $\delta(d \lambda)$ is singular, namely $\delta(\lambda)=\chi_{\Delta}(\lambda) F_{X}(d \lambda)$

$$
\Delta \subset(-\infty, \infty), L(\Delta)=0, \bar{\Delta}=(-\infty, \infty)-\Delta
$$

Let

$$
A_{t}=\left\{h: h=\int_{0}^{\infty} b(s) U^{-s} \gamma d s, y \in H_{X}(t)\right\}
$$

Obviously, At is linear set.

Lemma 1. Let $X(t), t \in \mathbf{R}$ is stationary processes, $F(d \lambda)$ and $Z(d \lambda)$ are spectral measure and random spectral measure respectively. $\forall f(\lambda), \phi(\lambda) \in L^{2}(F)$, and $|\phi(\lambda)| \leq M, M$ $>0$, where $M$ is real number, we have 


$$
\left\|\int_{-\infty}^{\infty} f(\lambda) \varphi(\lambda) Z(d \lambda)\right\| \leq M\left\|\int_{\infty}^{\infty} f(\lambda) Z(d \lambda)\right\|
$$

Proof. According to the nature of the random integral, we have

$$
\begin{aligned}
& \left\|\int_{-\infty}^{\infty} f(\lambda) \varphi(\lambda) Z(d \lambda)\right\| \\
= & {\left[\int_{-\infty}^{\infty}|f(\lambda) \varphi(\lambda)|^{2} F(d \lambda)\right]^{\frac{1}{2}} } \\
= & {\left[\int_{-\infty}^{\infty}|f(\lambda)|^{2} \cdot|\varphi(\lambda)|^{2} F(d \lambda)\right]^{\frac{1}{2}} } \\
\leq & M\left[\int_{-\infty}^{\infty}|f(\lambda)|^{2} \cdot F(d \lambda)\right]^{\frac{1}{2}} \\
= & M\left\|\int_{-\infty}^{\infty} f(\lambda) Z(d \lambda)\right\|
\end{aligned}
$$

\section{Lemma 2.}

$$
\mathcal{L}\left\{A_{t}\right\}=H_{\eta}(t)
$$

where $\mathcal{L}\left(A_{t}\right)$ is the linear closed manifold of $A_{t}$.

Proof. $\forall \eta\left(\tau_{0}\right), \tau_{0} \leq t$, Let $y=X\left(\tau_{0}\right)$, we have $y \in H_{X}(t)$, and

$$
\eta\left(\tau_{0}\right)=\int_{0}^{\infty} b(s) U^{-s} y d s=\int_{0}^{\infty} b(s) U^{-s} X\left(\tau_{0}\right) d s
$$

namely, $\eta\left(\tau_{0}\right) \in A_{t}$, then

$$
H_{\eta}(t) \subset \mathcal{L}\left(A_{t}\right)
$$

On the other hand, $\forall h \in A_{t}$, we have

$$
h=\int_{0}^{\infty} b(s) U^{-s} y d s
$$

where $y \in H_{X}(t)$.

Let $z_{l}=\sum_{k=1}^{m_{l}} a_{k}^{l} X\left(t_{k}^{l}\right), t_{k}^{l} \leq t, k=1,2, \ldots, m_{\mathrm{l}}, a_{k}^{l}$, is complex number. Let

$$
\left\|y-z_{l}\right\| \rightarrow 0(l \rightarrow \infty)
$$

while

$$
\int_{0}^{\infty} b(s) U^{-s} z_{l} d s=\sum_{k=1}^{m_{l}} a_{k}^{l} \int_{0}^{\infty} b(s) U^{-s} X\left(t_{k}^{l}\right) d s \in H_{\eta}(t), \quad l=1,2, \cdots
$$

Let, the spectral characteristics of $y$ and $z_{t}$ are $\psi_{y}(\lambda)$ and $\psi_{z_{l}}(\lambda)$ in the space $L^{2}\left(F_{X}\right.$ $(d \lambda))$ respectively. According to the equation (2-3) 


$$
|B(\lambda)|=\left|\int_{0}^{\infty} b(s) e^{-i \lambda s} d s\right| \leq \int_{0}^{\infty}|b(s)| d s \stackrel{\wedge}{=} M
$$

where $M>0$ is constant. According to the lemma 1 , we get

$$
\begin{aligned}
& \left\|h-\int_{0}^{\infty} b(s) U^{-s} z_{l} d s\right\| \\
= & \left\|\int_{0}^{\infty} b(s) U^{-s}\left(y-z_{l}\right) d s\right\| \\
= & \left\|\int_{0}^{\infty} b(s) \int_{-\infty}^{\infty} e^{-i s \lambda}\left(\psi_{y}(\lambda)-\psi_{z_{l}}(\lambda)\right) \Phi_{X}(d \lambda) d s\right\| \\
= & \left\|\int_{-\infty}^{\infty}\left(\psi_{y}(\lambda)-\psi_{z_{l}}(\lambda)\right) \int_{0}^{\infty} b(s) e^{-i s \lambda} d s \Phi_{X}(d \lambda)\right\| \\
= & \left\|\int_{-\infty}^{\infty}\left(\psi_{y}(\lambda)-\psi_{z_{l}}(\lambda)\right) B(\lambda) \Phi_{X}(d \lambda)\right\| \\
\leq & M\left\|\int_{-\infty}^{\infty}\left(\psi_{y}(\lambda)-\psi_{z_{l}}(\lambda)\right) \Phi_{X}(d \lambda)\right\| \\
= & M\left[\int_{-\infty}^{\infty}\left|\psi_{y}(\lambda)-\psi_{z_{l}}(\lambda)\right|^{2} F(d \lambda)\right]^{\frac{1}{2}} \\
= & M\left\|y-z_{l}\right\| \rightarrow 0(l \rightarrow \infty)
\end{aligned}
$$

so, $h \in H_{\eta}(t)$, namely

$$
\mathcal{L}\left\{A_{t}\right\} \subset H_{\eta}(t)
$$

Then, it shows the equation (3-3) is correct.

According to the lemma 2, we have

$$
\begin{aligned}
Q & =\inf _{\widetilde{\xi} \in H_{X}(t)}\left\|\int_{0}^{\infty} b(s) U^{-s} \widetilde{\xi} d s-\xi\right\|^{2} \\
& =\inf _{h \in A_{t}}\|h-\xi\|^{2} \\
& =\inf _{h \in L\left\{A_{t}\right\}}\|h-\xi\|^{2} \\
& =\inf _{h \in H_{\eta}(t)}\|h-\xi\|^{2} \\
& =\left\|P_{H_{\eta}(t)} \xi-\xi\right\|^{2}
\end{aligned}
$$

According to the equation (2-5) and (2-1), we get

$$
\begin{aligned}
\eta(t) & =\int_{0}^{\infty} b(s) X(t-s) d s=\int_{0}^{\infty} b(s) \int_{-\infty}^{\infty} e^{i(t-s) \lambda} \Phi_{X}(d \lambda) d s \\
& =\int_{-\infty}^{\infty} e^{i t \lambda}\left[\int_{0}^{\infty} b(s) e^{-i s \lambda} d s\right] \Phi_{X}(d \lambda)=\int_{-\infty}^{\infty} e^{i t \lambda} B(\lambda) \Phi_{X}(d \lambda)
\end{aligned}
$$

on the other hand

$$
\eta(t)=\int_{-\infty}^{\infty} e^{i t \lambda} \Phi_{\eta}(d \lambda)
$$

According to the stochastic process spectral theorem and the Relevant function spectral theorem, we have 


$$
\begin{aligned}
& \Phi_{\eta}(d \lambda)=B(\lambda) \Phi_{X}(d \lambda) \\
& F_{\eta}(d \lambda)=|B(\lambda)|^{2} F_{X}(d \lambda) \\
& f_{\eta}(d \lambda)=|B(\lambda)|^{2} f_{X}(\lambda)
\end{aligned}
$$

where $\Phi_{\eta}(d \lambda), F_{\eta}(d \lambda), f_{\eta}(\lambda)$ representative the random spectral measure, spectral measure and broad spectral measure of $\eta(t)$.

Lemma 3. Let $\xi \in H_{X}, \xi=\widehat{\xi}+\widetilde{\xi}$, where $\widehat{\xi} \in H_{\eta}, \widetilde{\xi} \perp H_{\eta}$, then

$$
Q=\|\widetilde{\xi}\|^{2}+\left\|P_{H_{\eta}(t)} \widehat{\xi}-\widehat{\xi}\right\|^{2}
$$

If, the wold decomposition of $\eta(t)$ is

$$
\eta(t)=\eta^{r}(t)+\eta^{s}(t)
$$

and

$$
\xi=\xi^{r}+\xi^{s}
$$

where $\eta^{r}$ is regular process, $\eta^{s}(t)$ is singular process, and $\widehat{\xi}^{r} \perp S_{\eta^{\prime}} \widehat{\xi}^{s} \in S_{\eta^{\prime}}$ $S_{\eta}=\bigcap_{t} H_{\eta}(t)$, then

$$
Q=\|\widetilde{\xi}\|^{2}+\left\|P_{H_{\eta} r(t)} \widehat{\xi}^{r}-\widehat{\xi}^{r}\right\|^{2}
$$

Proof. According to $\xi=\widehat{\xi}+\widetilde{\xi}, \widehat{\xi} \in H_{\eta}, \widetilde{\xi} \perp H_{\eta}$, So

$$
P_{H_{\eta}(t)} \xi=P_{H_{\eta}(t)} \widehat{\xi}+P_{H_{\eta}(t)} \widetilde{\xi}=P_{H_{\eta}(t)} \widehat{\xi}
$$

According to the equation (3-4),

$$
\begin{aligned}
Q & =\left\|P_{H_{\eta}(t)} \xi-\xi\right\|^{2}=\left\|P_{H_{\eta}(t)} \xi-(\widehat{\xi}+\widetilde{\xi})\right\|^{2} \\
& =\left\|\left(P_{H_{\eta}(t)} \widehat{\xi}-\widehat{\xi}\right)-\widetilde{\xi}\right\|^{2}
\end{aligned}
$$

also, according to $\widetilde{\xi} \perp\left(P_{H_{\eta}(t)} \widehat{\xi}-\widehat{\xi}\right)$, So

$$
Q=\|\tilde{\xi}\|^{2}+\left\|P_{H_{\eta}(t)} \widehat{\xi}-\widehat{\xi}\right\|^{2}
$$

namely, the equation (3-8) is correct.

When $\eta(t)$ have the wold decomposition, notice

$$
\widehat{\xi}^{S}=P_{H_{\eta}} \widehat{\xi}, H_{\eta}(t)=H_{\eta^{r}}(t) \oplus S_{\eta}
$$

we get

$$
\begin{aligned}
& P_{H_{\eta}(t)} \widehat{\xi}^{s}=P_{H_{\eta}(t)}\left(P_{S_{\eta}} \widehat{\xi}\right)=P_{S_{\eta}} \widehat{\xi}=\widehat{\xi}^{s} \\
& P_{H_{\eta}(t)} \widehat{\xi}^{r}=P_{H_{\eta^{r}}(t)} \widehat{\xi}^{r}+P_{S_{\eta}} \widehat{\xi}^{r}=P_{H_{\eta^{r}}(t)} \widehat{\xi}^{r}
\end{aligned}
$$

So

$$
\begin{aligned}
& \left\|P_{H_{\eta}(t)} \widehat{\xi}-\widehat{\xi}\right\|^{2} \\
= & \|\left(P_{H_{\eta}(t)} \widehat{\xi}^{r}+P_{H_{\eta}(t)} \widehat{\xi}^{s}-\left(\widehat{\xi}^{r}+\widehat{\xi}^{s}\right) \|^{2}\right. \\
= & \left\|P_{H_{\eta^{r}}(t)} \widehat{\xi}^{r}-\widehat{\xi}^{r}\right\|^{2}
\end{aligned}
$$


Then, according to the equation (3-8), we get that the equation (3-9) is correct.

Lemma 4. Let $\xi \in H_{X}, \xi=\widehat{\xi}+\widetilde{\xi}$, where $\widehat{\xi} \in H_{\eta}, \widetilde{\xi} \perp H_{\eta}, \psi(\lambda)$ is spectral characteristics of $\xi$ in the space of $L^{2}\left(F_{X}(d \lambda)\right), \widehat{\psi}(\lambda)$ is spectral characteristics of $\widehat{\xi}$ in the space of $L^{2}$ $\left(F_{\eta}(d \lambda)\right)$, then

1) when $\lambda \in \bar{E}$,

$$
\psi(\lambda)=\widehat{\psi}(\lambda) B(\lambda), \quad \text { a.e. } \quad F_{X}(d \lambda)
$$

2)

$$
\|\tilde{\xi}\|^{2}=\int_{E}|\psi(\lambda)|^{2} F_{X}(d \lambda)
$$

Proof. 1) According to the given conditions, we have

$$
\begin{aligned}
& \xi=\int_{-\infty}^{\infty} \psi(\lambda) \Phi_{X}(d \lambda) \\
& \widehat{\xi}=\int_{-\infty}^{\infty} \widehat{\psi}(\lambda) \Phi_{\eta}(d \lambda)=\int_{-\infty}^{\infty} \widehat{\psi}(\lambda) B(\lambda) \Phi_{X}(d \lambda) \\
& \eta(t)=\int_{-\infty}^{\infty} e^{i t \lambda} \Phi_{\eta}(d \lambda)=\int_{-\infty}^{\infty} e^{i t \lambda} B(\lambda) \Phi_{X}(d \lambda)
\end{aligned}
$$

So

$(\xi, \eta(-t))=\int_{-\infty}^{\infty} e^{i t \lambda} \psi(\lambda) \overline{B(\lambda)} F_{X}(d \lambda)$

on the other hand

$$
\begin{aligned}
(\xi, \eta(-t)) & =(\widehat{\xi}+\widetilde{\xi}, \eta(-t))=(\widehat{\xi}, \eta(-t)) \\
& =\int_{-\infty}^{\infty} e^{i t \lambda} \widehat{\psi}(\lambda)|B(\lambda)|^{2} F_{X}(d \lambda)
\end{aligned}
$$

According to the Fourier transformation, we have

$$
\psi(\lambda) \overline{B(\lambda)}=\widehat{\psi}(\lambda)|B(\lambda)|^{2}, \quad \text { a.e. } \quad F_{X}(d \lambda)
$$

So, when $\lambda \in \bar{E}$, we have

$$
\psi(\lambda)=\widehat{\psi}(\lambda) B(\lambda), \quad \text { a.e. } \quad F_{X}(d \lambda)
$$

2) According to $\xi=\widehat{\xi}+\widetilde{\xi}$, and $\widehat{\xi} \perp \widetilde{\xi}$, Thus

$$
\begin{aligned}
\|\xi\|^{2} & =\left|\widehat{\xi}+\widetilde{\xi}\left\|^{2}=\right\| \widehat{\xi}\left\|\left.\right|^{2}+\right\| \tilde{\xi} \|^{2}\right. \\
\|\tilde{\xi}\|^{2} & =\|\xi\|^{2}-\|\widehat{\xi}\|^{2} \\
& =\int_{-\infty}^{\infty}|\psi(\lambda)|^{2} F_{X}(d \lambda)-\int_{-\infty}^{\infty}|\widehat{\psi}(\lambda)|^{2} F_{\eta}(d \lambda) \\
& =\int_{-\infty}^{\infty}|\psi(\lambda)|^{2} F_{X}(d \lambda)-\int_{-\infty}^{\infty}|\widehat{\psi}(\lambda)| \cdot|B(\lambda)|^{2} F_{X}(d \lambda) \\
& =\int_{-\infty}^{\infty} \chi_{E}(\lambda)|\psi(\lambda)|^{2} F_{X}(d \lambda)+\int_{-\infty}^{\infty} \chi_{\bar{E}}(\lambda)|\psi(\lambda)|^{2} F_{X}(d \lambda)-\int_{-\infty}^{\infty} \chi_{\bar{E}}(\lambda)|\widehat{\psi}(\lambda)|^{2} \cdot|B(\lambda)|^{2} F_{X}(d \lambda) \\
& =\int_{-\infty}^{\infty} \chi_{E}(\lambda)|\psi(\lambda)|^{2} F_{X}(d \lambda)=\int_{E}|\psi(\lambda)|^{2} F_{X}(d \lambda)
\end{aligned}
$$


namely, the equation (3-13) is correct.

Theorem. Let $\xi \in H_{X}, \xi=\widehat{\xi}+\widetilde{\xi}$, where $\widehat{\xi} \in H_{\eta}, \widetilde{\xi} \perp H_{\eta}, \psi(\lambda)$ representative the spectral characteristics of $\xi$ in the space of $L^{2}\left(F_{X}(d \lambda)\right), \widehat{\psi}(\lambda)$ representative the spectral characteristics of $\widehat{\xi}$ in the space of $L^{2}\left(F_{\eta}(d \lambda)\right)$. Then

1) When $\frac{\log f_{x}(\lambda)}{1+\lambda^{2}} \notin L^{1}(-\infty, \infty)$

$$
Q=\int_{E}|\psi(\lambda)|^{2} F_{X}(d \lambda)
$$

now the spectral characteristics of $P_{H_{\eta}(t)} \xi$ in the space of $L^{2}\left(F_{X}(d \lambda)\right)$ is

$$
\widetilde{\psi}(\lambda)=\left\{\begin{array}{l}
0, \quad \lambda \in E, \\
\psi(\lambda), \lambda \in \bar{E} .
\end{array}\right.
$$

2) When $\frac{\log f_{X}(\lambda)}{1+\lambda^{2}} \in L^{1}(-\infty, \infty)$

$$
Q=\int_{E}|\psi(\lambda)|^{2} F_{X}(d \lambda)+\int_{t}^{\infty}|\varphi(-s)|^{2} d s
$$

now the spectral characteristics of $P_{H_{\eta}(t)} \xi$ in the space of $L^{2}\left(F_{X}(d \lambda)\right)$ is

$$
\widetilde{\psi}(\lambda)= \begin{cases}0, & \lambda \in E, \\ \psi(\lambda), & \lambda \in \bar{E} \Delta, \\ \frac{B(\lambda) \int_{-t}^{\infty}}{\Gamma(\lambda)}, & \lambda \in \overline{E \Delta}\end{cases}
$$

where $b(t), B(\lambda), \eta(t), E$ and $\Delta$ is decided by the equation (2-1), (2-2), (2-3), (2-5), (2$4)$ and (3-1) respectively. Where $\Gamma(\lambda)$ is the maximal factor boundary values of the spectral density $f_{\eta}(\lambda), \phi(\lambda)$ is the Fourier transformation of $\widehat{\psi}(\lambda) \Gamma(\lambda)$, where $\widehat{\psi}(\lambda)$ is determined by equation (3-12) and $\widehat{\psi}(\lambda)=\psi(\lambda) / B(\lambda)$, a.e. $L$.

Proof. 1) When $\frac{\log f_{x}(\lambda)}{1+\lambda^{2}} \notin L^{1}(-\infty, \infty)$. According to

$$
\begin{aligned}
& f_{\eta}(\lambda)=|B(\lambda)|^{2} f_{X}(\lambda) \\
& \log |B(\lambda)| \in L^{1}(-\infty, \infty)
\end{aligned}
$$

we know that

$$
\frac{\log f_{\eta}(\lambda)}{1+\lambda^{2}} \notin L^{1}(-\infty, \infty)
$$

Thus, $\eta(t)$ is singular process. So

$$
\begin{gathered}
H_{\eta}=S_{\eta} \\
P_{H_{\eta}(t)} \widehat{\xi}=P_{H_{\eta}} \widehat{\xi}=P_{S_{\eta}} \widehat{\xi}=\widehat{\xi}
\end{gathered}
$$

According to (3-8)

$$
Q=\|\tilde{\xi}\|^{2}=\int_{E}|\psi(\lambda)|^{2} F_{X}(d \lambda) .
$$


On the other hand

$$
P_{H_{\eta}(t)} \xi=P_{H_{\eta}}(\widehat{\xi}+\widetilde{\xi})=P_{H_{\eta}} \widehat{\xi}=P_{S_{\eta}} \widehat{\xi}=\widehat{\xi}
$$

The spectral characteristics of $P_{H_{\eta}(t)} \xi$ in the space $L^{2}\left(F_{X}(d \lambda)\right)$ is

$$
\widetilde{\psi}(\lambda)=\widehat{\psi}(\lambda) B(\lambda), \quad \text { a.e. } \quad F_{X}(d \lambda)
$$

According to the equation (3-12), we get

$$
\widetilde{\psi}(\lambda)=\left\{\begin{array}{l}
0, \quad \lambda \in E_{,} \\
\psi(\lambda), \lambda \in \bar{E},
\end{array} \text { a.e. } F_{X}(d \lambda)\right.
$$

2) When $\frac{\log f_{X}(\lambda)}{1+\lambda^{2}} \in L^{1}(-\infty, \infty)$, according to (3-18) and (3-19), we get $\frac{\log f_{\eta}(\lambda)}{1+\lambda^{2}} \in L^{1}(-\infty, \infty)$.

It shows that $\eta(t)$ is non-singular process, so $\eta(t)$ has regular singular decomposition, and it consistent with Lebesgue-Gramer decomposition. Let the decomposition equation is

$$
\eta(t)=\eta^{r}(t)+\eta^{s}(t)
$$

where $\eta^{r}(t)$ is regular process, $\eta^{s}(t)$ is singular process

$$
\begin{aligned}
& \eta^{r}(t)=\int_{-\infty}^{\infty} e^{i t \lambda} \Phi_{\eta^{r}}(d \lambda)=\int_{-\infty}^{\infty} e^{i t \lambda} \chi_{\bar{\Delta}}(\lambda) \Phi_{\eta}(d \lambda) \\
& \eta^{s}(t)=\int_{-\infty}^{\infty} e^{i t \lambda} \Phi_{\eta^{s}}(d \lambda)=\int_{-\infty}^{\infty} e^{i t \lambda} \chi_{\Delta}(\lambda) \Phi_{\eta}(d \lambda)
\end{aligned}
$$

Let $V(d s)$ is basic cross stochastic measure, namely

$$
V\left(\Delta_{1}\right)=\int_{-\infty}^{\infty} \frac{e^{i \lambda t_{2}}-e^{i \lambda t_{1}}}{i \lambda} \Lambda(d \lambda)
$$

where $\Delta_{1}=\left(t_{1}, t_{2}\right]$, stochastic measure $\Lambda(d \lambda)=\frac{1}{\Gamma(\lambda)} \Phi_{\eta^{r}}(d \lambda)$, Thus

$$
\eta^{r}(t)=\int_{-\infty}^{t} C(t-s) V(d s)
$$

when $C(s)=\frac{1}{2 \pi} \int_{-\infty}^{\infty} e^{i s \lambda} \Gamma(\lambda) d \lambda$, and $\Gamma(\lambda)$ satisfate the follow conditions

$$
f(\lambda)=\frac{1}{2 \pi}|\Gamma(\lambda)|^{2}
$$

also $\Gamma(\lambda)$ is the boundary values of the lower half plane maximum analytic functions $\Gamma(z)$, notice

$$
\begin{aligned}
\widehat{\xi}^{r} & =\int_{-\infty}^{\infty} \widehat{\psi}(\lambda) \chi_{\bar{\Delta}}(\lambda) \Phi_{\eta}(d \lambda)=\int_{-\infty}^{\infty} \widehat{\psi}(\lambda) \Phi_{\eta^{r}}(d \lambda) \\
& =\int_{-\infty}^{\infty} \widehat{\psi}(\lambda) \Gamma(\lambda) \Lambda(d \lambda) \\
\widehat{\xi}^{s} & =\int_{-\infty}^{\infty} \widehat{\psi}(\lambda) \chi_{\Delta}(\lambda) \Phi_{\eta}(d \lambda)=\int_{-\infty}^{\infty} \widehat{\psi}(\lambda) \Phi_{\eta^{s}}(d \lambda)
\end{aligned}
$$


Let $\phi(s)$ is the Fourier transforation of $\widehat{\psi}(\lambda) \Gamma(\lambda)$, namely

$$
\varphi(s)=\frac{1}{2 \pi} \int_{-\infty}^{\infty} e^{i s \lambda} \widehat{\psi}(\lambda) \Gamma(\lambda) d \lambda
$$

According to the Fourier transformation of random measure

$$
\begin{aligned}
\widehat{\xi}^{r} & =\int_{-\infty}^{\infty} \widehat{\psi}(\lambda) \Gamma(\lambda) \Lambda(d \lambda)=\int_{-\infty}^{\infty} \int_{-\infty}^{\infty} e^{-i \lambda s} \varphi(s) d s \Lambda(d \lambda) \\
& =\int_{-\infty}^{\infty} \int_{-\infty}^{\infty} e^{i \lambda s} \varphi(-s) d s \Lambda(d \lambda)=\int_{-\infty}^{\infty} \varphi(-s) V(d s)
\end{aligned}
$$

Thus

$$
\begin{gathered}
P_{H_{\eta^{r}}(t)} \hat{\xi}^{r}=\int_{-\infty}^{t} \varphi(-s) V(d s) \\
\left\|P_{H_{\eta^{r}}(t)} \hat{\xi}^{r}-\hat{\xi}^{r}\right\|^{2}=\left\|\int_{t}^{\infty} \varphi(-s) V(d s)\right\|^{2}=\int_{t}^{\infty}|\varphi(-s)|^{2} d s
\end{gathered}
$$

According to the equation (3-9), we get

$$
Q=\int_{E}|\psi(\lambda)|^{2} F_{X}(d \lambda)+\int_{t}^{\infty}|\varphi(-s)|^{2} d s
$$

According to the Lemma 3

$$
P_{H_{\eta}(t)} \xi=P_{H_{\eta}(t)} \widehat{\xi}=P_{H_{\eta}(t)} \widehat{\xi}^{r}+P_{H_{\eta}(t)} \widehat{\xi}^{s}=P_{H_{\eta^{r}}(t)} \widehat{\xi}^{r}+\widehat{\xi}^{s}
$$

notice

$$
\begin{aligned}
P_{H_{\eta^{r}}(t)} \widehat{\xi}^{r} & =\int_{-\infty}^{t} \varphi(-s) V(d s)=\int_{-\infty}^{\infty}\left[\int_{-\infty}^{t} e^{i \lambda s} \varphi(-s) d s\right] \Lambda(d \lambda) \\
& =\int_{-\infty}^{\infty}\left[\int_{-t}^{\infty} e^{-i \lambda s} \varphi(s) d s\right] \frac{1}{\Gamma(\lambda)} \Phi_{\eta^{r}}(d \lambda) \\
& =\int_{-\infty}^{\infty}\left[\int_{-t}^{\infty} e^{-i \lambda s} \varphi(s) d s\right] \frac{1}{\Gamma(\lambda)} \chi_{\bar{\Delta}}(\lambda) B(\lambda) \Phi_{X}(d \lambda) \\
\widehat{\xi}^{s} & =\int_{-\infty}^{\infty} \widehat{\psi}(\lambda) \chi_{\Delta}(\lambda) \Phi_{\eta}(d \lambda)=\int_{-\infty}^{\infty} \widehat{\psi}(\lambda) \chi_{\Delta}(\lambda) B(\lambda) \Phi_{X}(d \lambda)
\end{aligned}
$$

Thus

$$
P_{H_{\eta}(t)} \xi=\int_{-\infty}^{\infty}\left[\int_{-t}^{\infty} e^{-i \lambda s} \varphi(s) d s \frac{B(\lambda)}{\Gamma(\lambda)} \chi_{\Delta}(\lambda)+\widehat{\psi}(\lambda) B(\lambda) \chi_{\Delta}(\lambda)\right] \Phi_{X}(d \lambda)
$$

So, the spectral characteristics of $P_{H_{\eta}(t)} \xi$ in the space of $L^{2}\left(F_{X}(d \lambda)\right)$ is

$$
\widetilde{\psi}(\lambda)= \begin{cases}0, & \lambda \in E \\ \psi(\lambda), & \lambda \in \bar{E} \Delta \\ \frac{B(\lambda) \int_{-t}^{\infty} e^{-i \lambda s} \varphi(s) d s}{\Gamma(\lambda)}, & \lambda \in \overline{E \Delta}\end{cases}
$$

\section{Conclusions}

A fixed transform is given which based on one-dimensional a stationary processes in this paper. Also, we propose a general filtering problem. Then, in the space of $L^{2}\left(F_{X}\right.$ $(d \lambda)$ ), we get the spectral characteristics of $P_{H_{\eta}(t)} \xi$ with no any additional conditions. Finally, we calculate the value of the best predict quantity of the general filtering problem. 


\section{Acknowledgements}

This work was supported by the Natural Science Foundation of China (Grant no. 10771047).

\section{Authors' contributions}

The studies and manuscript of this paper was written by Longsuo Li independently.

\section{Competing interests}

The author declares that they have no competing interests.

Received: 5 May 2011 Accepted: 23 September 2011 Published: 23 September 2011

\section{References}

1. Rosanov, A IV: The spectral theory of multidimensional stationary random processes with discrete time. Uspekhi Mat. Nauk. Z3 93-142 (1958). (in Russian)

2. Gong, ZR, Zhu, YS, Cheng, QS: Optimum filter problem of random signal for a linear system. Journal of Harbin University of Science and Technology., 1 (1981)

3. Gong, ZR, Tong, GR, Cheng, QS: On filtering problem of multidimensional stationary sequence for a linear system. Journal of Wuhan University (Natural Science., 2 (1982)

4. Zhu, YS, Gong, ZR: General filter problem of multidimensional stationary sequence for a linear system. Mathematical Theory and Applied Probability. 5(1) (1990)

5. Zhang, DC: Solution of linear prediction problems with fixed transformation in stationary stochastic processes. Journal of Huazhong University of Science and Technology(Nature Science Edition). 9(2) (1981)

6. Zhang, $X Y, X i e, Z$ J: On the extrapolation of multivariate stationary time series for a linear system. Journal of Henan Normal University (Natural Science)., 3 (1983)

7. Zhang, $X Y, X i e, Z$ J: On the extrapolation of multivariate stationary time series for a linear system. Journal of Henan Normal University(Natural Science)., 4 (1983)

8. Xie, ZJ, Cheng, QS: On the extrapolation of stationary time series for a linear system. Fifth European Meating on Cybesneties and Systems Research(edited by austrian society for cybernetie studies). (1980)

9. Zhang, XY: A few problems in the extrapolation of multivariate stationary time series for a linear system. Journal of Henan Normal University(Natural Science)., 4 (1984)

doi:10.1186/1029-242X-2011-68

Cite this article as: Li: On general filtering problem of stationary processes with fixed transformation. Journal of Inequalities and Applications 2011 2011:68.

\section{Submit your manuscript to a SpringerOpen ${ }^{\circ}$ journal and benefit from:}

- Convenient online submission

- Rigorous peer review

- Immediate publication on acceptance

- Open access: articles freely available online

- High visibility within the field

- Retaining the copyright to your article

Submit your next manuscript at $\boldsymbol{\nabla}$ springeropen.com 